\title{
Labor induction in preeclampsia: Is misoprostol more effective than dinoprostone?
}

\author{
Olav Lapaire, Rosanna Zanetti-Dällenbach, \\ Patrizia Weber, Irene Hösli, Wolfgang \\ Holzgreve and Daniel Surbek* \\ Department of Obstetrics and Gynecology, University \\ Hospital Basel, Switzerland
}

\begin{abstract}
Objective: To compare the efficacy of vaginal misoprostol versus dinoprostone for induction of labor (IOL) in patients with preeclampsia according to the WHO criteria.

Study design: Ninety-eight patients were retrospectively analyzed. A total of 47 patients received $3 \mathrm{mg}$ dinoprostone suppositories every $6 \mathrm{~h}$ ( $\max .6 \mathrm{mg} / 24 \mathrm{~h}$ ) whereas 51 patients in the misoprostol group received either $50 \mu \mathrm{g}$ misoprostol vaginally every $12 \mathrm{~h}$, or $25 \mu \mathrm{g}$ every $6 \mathrm{~h}$ (max. $100 \mu \mathrm{g} / 24 \mathrm{~h}$ ). Primary outcomes were vaginal delivery within 24 and $48 \mathrm{~h}$, respectively.

Results: The probability of delivering within $48 \mathrm{~h}$ was more than three-fold higher in the misoprostol than in the dinoprostone group: odds ratio $(\mathrm{OR})=3.48$; 95\% confidence interval $(\mathrm{Cl}) 1.24,10.30$, whereas no significant difference was observed within $24 \mathrm{~h}(\mathrm{P}=0.34)$. No correlation was seen between a ripe cervix prior to IOL and delivery within $24 / 48 \mathrm{~h}(\mathrm{P}=0.33$ and $\mathrm{P}=1.0$, respectively). More cesarean sections were performed in the dinoprostone group due to failed IOL $(P=0.0009)$. No significant differences in adverse maternal outcome were observed between both study groups, whereas more neonates (12 vs. 6) of the dinoprostone group were admitted to the NICU $(P=0.068)$.

Conclusion: This study suggests that misoprostol may have some advantages compared to dinoprostone, including improved efficacy and lower cost of the drug, even in cases of preeclampsia.
\end{abstract}

\footnotetext{
*Corresponding author:

Prof. Daniel Surbek, MD

Department of Obstetrics and Gynecology

University Hospital

Effingerstrasse 102

$\mathrm{CH}-3010$ Berne

Switzerland

Tel.: +4131632 1103

Fax: +41316321105

E-mail: daniel.surbek@insel.ch
}

Keywords: Dinoprostone; induction of labor; misoprostol; preeclampsia.

\section{Introduction}

Preeclampsia complicates approximately 5\% of all pregnancies and is associated with significant morbidity and mortality for both the pregnant patient and her fetus [15]. Furthermore, the only way to arrest disease progression is to deliver the fetus and placenta. Therefore, induction of labor (IOL) in case of mild or moderate preeclampsia is often the method of choice, also intended to avoid cesarean section associated complications. IOL has become a routine procedure in obstetrical management, especially in developed countries, currently affecting more than $20 \%$ of all deliveries in the United States (US) [8]. The success of IOL may not only be dependent on the ripeness of the cervix, but also on the inducing agent. In the case of an unripe cervix, the use of prostaglandins, introduced in the 1960s for cervical ripening, is approved as first-line treatment. Prostaglandin (PG) E2 is currently the only regimen for cervical ripening that has been approved in the US by the U.S. Food and Drug Administration (FDA), whereas misoprostol (PGE1), recommended by the ACOG as an alternative agent for IOL in women without a previous uterine scar, is considered to be an off-label indication [1]. Advantages of PGE1 include high efficacy, low cost and easy storage conditions. Misoprostol showed a higher efficacy than other vaginal or intracervical prostaglandins for IOL in the case of non-preeclamptic patients [7, 16].

However, there exist only few trials that investigated the effectiveness of both agents, specifically in the case of preeclampsia [5, 9, 10, 12, 22]. Furthermore, induction of labor often may be challenging in the case of preeclampsia due to generally unfavorable Bishop scores, lower gestational age and a high proportion of nulliparity and intravenously applicated magnesium, which may have a tocolytic effect [10]. In preeclampsia it is particularly important to aim for deliveries within $24-48 \mathrm{~h}$, as the condition has a tendency to worsen with time. In the present study we aimed to determine the effectiveness of vaginal misoprostol for labor induction in preeclampsia as compared with dinoprostone in a single tertiary center. Primary outcomes were vaginal delivery within 24 and 
$48 \mathrm{~h}$. Secondary outcome measures included the induction to delivery interval, maternal and fetal outcome, mode of delivery and drug related side effects.

\section{Methods}

This retrospective study was conducted at one tertiary center. A total of ninety-eight patients (51 in the misoprostol and 47 in the dinoprostone groups) were identified from the database of the Women's University Hospital of Basel between the period of January 1990-December 1996 (dinoprostone group) and January 1999-December 2002 (misoprostol group). In those two time periods, dinoprostone and subsequently misoprostol represented the first line agent for IOL, taking the exclusion criteria into account (e.g., hypersensibility to prostaglandins, previous cesarean section in case of misoprostol). The inclusion criteria consisted of induced patients with preeclampsia, according to the WHO criteria [hypertension $>140 / 90 \mathrm{~mm} \mathrm{Hg}$, increase of the systolic or diastolic blood pressure $>30 \mathrm{~mm} \mathrm{Hg}$ and $15 \mathrm{~mm} \mathrm{Hg}$, respectively, on two occasions at least $6 \mathrm{~h}$ apart, in combination with proteinuria (>300 mg/24 h)], singleton fetuses in vertex presentation, intact amniotic membranes and absence of active labor. Exclusion criteria were: previous uterine surgery, placenta previa, fetal anomaly, known allergy to prostaglandins, maternal age $<18$ years and pathologic non-stress cardiotocogram prior to induction. The patients received either misoprostol (PG E1) in a dose regimen of $25 \mu \mathrm{g}$ vaginally every $6 \mathrm{~h}$, or $50 \mu \mathrm{g}$ vaginally every $12 \mathrm{~h}$. The maximum dose for all women was $100 \mu \mathrm{g} / 24 \mathrm{~h}$. The patients in the dinoprostone group received $3 \mathrm{mg}$ dinoprostone suppositories with a 6-h time interval (maximum of $6 \mathrm{mg} /$ $24 \mathrm{~h}$ ). External electronic fetal monitoring was performed $30 \mathrm{~min}$ before and $120 \mathrm{~min}$ after each medication was administered. If there were no contractions, 6 or $12 \mathrm{~h}$ after the first dose, dependent on the dose regimen, each patient was digitally reassessed. When the Bishop score was $\leq 8$, a second dose of dinoprostone or misoprostol was administered, according to the guidelines. The procedure was repeated the following day. If progress towards delivery did not occur after $48 \mathrm{~h}$ or if the underlying disease had progressed, the patient was re-evaluated for further management. No further dose of either medication was given if there were uterine contractions, if the membranes ruptured spontaneously or if there were fetal heart rate abnormalities. The subsequent management of labor was identical for both groups. In case of failure of cervical dilatation $(<1 \mathrm{~cm} / \mathrm{h})$ or no progress during the active first and second stage of labor, intravenous oxytocin by infusion pump, at least six hours apart from the last $P G$ application, was initiated with increasing doses from 1.25 up to $20 \mathrm{mU} / \mathrm{min}$. Surveillance of fetal heart rate (FHR) and uterine activity were monitored in all patients. FHR patterns were analyzed according to the RCOG guideline [2]. In the case of severe preeclampsia (onset prior to 34 weeks, indicated by proteinuria $>5 \mathrm{~g} / 24 \mathrm{~h}$, hypertension $>160 / 110 \mathrm{~mm} \mathrm{Hg}$ ), or clinical signs (visual disturbances, hyperreflexia, severe headache), an anticonvulsive prophylaxis with intravenous magnesium sulfate was initiated by a $4 \mathrm{~g}$ bolus over $20 \mathrm{~min}$, followed by a continuous therapy of $2 \mathrm{~g} / \mathrm{h}$, according to quantitative measurements, until $48 \mathrm{~h}$ postpartum. All data were collected from the hospital records. The assessment of the cardiotocograms for uterine contraction abnormalities and abnormal fetal heart rate pattern was performed during labor and recorded in the patient's chart. The endpoint chosen to evaluate the effectiveness of misoprostol and dinoprostone were the achievement of a vaginal delivery within 24 and $48 \mathrm{~h}$, respectively. The sample size was calculated using the power analysis from a previous study of patients without preeclampsia to detect a $35 \%$ difference in the number of patients delivered within $24 \mathrm{~h}$ [16]. Thus, 48 patients in each group were required for the study to have a power of $80 \%$ at a type I error level of 0.05 .

\section{Statistical analysis}

To compare approximate normally distributed data in the groups, Student's $t$-test was used and mean with standard deviation was calculated. Data were logarithmically transformed if necessary.

Mann-Whitney $U$-test was used for ordinal data and median with range was tabulated. To describe associations in cross tabulations, Fisher exact test was performed and odds ratios with corresponding 95\% confidence intervals were reported. A $P$-value $<0.05$ was considered significant. As this was an exploratory study we did not adjust the P-values for multiple comparisons. All analyses were performed using SPSS (SPSS Inc., Chicago, USA) 11.5.1.

\section{Results}

Both groups were comparable in terms of demographic and obstetric data, such as maternal age, gravidity, parity, gestational age and birth weight (Tables 1 and 2). The Bishop scores collected prior to IOL were not statistically different in both groups $(P=0.33$; Table 1). No correlation was seen between a ripe cervix (Bishop score $>6$ ) and delivery within $24 \mathrm{~h}$ between both groups [odds ratio $(\mathrm{OR})=0.33$; (95\% confidence interval $(\mathrm{Cl}) 0.026-3.18)]$, or within $48 \mathrm{~h}[\mathrm{OR}=0.85$; $(95 \% \mathrm{Cl} 0.07-7.96)$, respectively]. A total of $78 \%(40 / 51)$ in the misoprostol group delivered spontaneously or by vaginal operative delivery as compared to $64 \%(30 / 47)$ in the dinoprostone group $(P=0.123)$. The rate of cesarean section (CS) did not differ between the groups $(P=0.12)$; however, $C S$ was required due to failure of induction in none (misoprostol) versus 11 patients in the dinoprostone group $(P=0.0009)$.

The type of PG administered for ripening had no significant influence on the mean induction to delivery time interval $(P=0.2$; Table 2). However, a statisticaly significant difference in the frequency of delivery within $48 \mathrm{~h}$ was observed: 30 patients in the misoprostol group versus 13 in the dinoprosone group delivered vaginally within $48 \mathrm{~h}(P=0.01)$. In contrast, the rate of vaginal deliveries within $24 \mathrm{~h}$ was not statistically different between both groups (19 patients in the misoprostol group, nine patients in the dinoprostone group, $\mathrm{P}=0.15$ ). The probability of a vaginal delivery within $48 \mathrm{~h}$ was more than three-fold higher with misoprostol than with dinoprostone $(\mathrm{OR}=3.48$; 95\% Cl: 1.23-10.30), whereas no significant difference was seen in vaginal deliveries within $24 \mathrm{~h}$ between both groups (OR=2.09; 95\% Cl: 0.72-6.38).

The dose of oxytocin administered during labor did not differ statistically between both groups $(P=0.34)$. Fur- 
Table 1 Population demographic and obstetric characteristics of the two groups.

\begin{tabular}{|c|c|c|c|}
\hline \multirow[t]{2}{*}{ Variable } & \multicolumn{2}{|l|}{ Drugs } & \multirow[t]{2}{*}{$\mathrm{P}$} \\
\hline & $\begin{array}{l}\text { Misoprostol } \\
\mathrm{n}=51\end{array}$ & $\begin{array}{l}\text { Dinoprostone } \\
\mathrm{n}=47\end{array}$ & \\
\hline Maternal age (years) (SD) & $29.31(5.4)$ & $28.49(4.8)$ & $0.43^{*}$ \\
\hline $\begin{array}{l}\text { Gestational age at delivery (weeks) } \\
\text { (range) (SD) }\end{array}$ & $\begin{array}{l}39.11 \\
(34-42)(2.8)\end{array}$ & $\begin{array}{l}38.34 \\
(32-42)(2.1)\end{array}$ & $0.49^{\star *}$ \\
\hline Gravidity (SD) & $1.55(1.0)$ & $1.94(1.5)$ & $0.14^{\star \star}$ \\
\hline Nulliparity (n (\%)) & $38(74.5)$ & $34(72.3)$ & $0.82^{\star \star \star}$ \\
\hline Bishop score (SD) & $2.93(1.4)$ & $3.27(1.8)$ & $0.34^{*}$ \\
\hline Magnesium sulfate intravenously (\%) & $8(15.7)$ & $10(21.2)$ & $0.60^{\star \star \star}$ \\
\hline Vaginal delivery (n (\%)) & $40(78.4)$ & $30(63.8)$ & $0.12^{\star \star \star}$ \\
\hline \multicolumn{4}{|l|}{ Spontaneous/vaginal operative } \\
\hline $\begin{array}{l}\text { Cesarean section due to failure of } \\
\text { induction of labor (n) }\end{array}$ & 0 & 9 & $0.0009^{\star \star *}$ \\
\hline
\end{tabular}

Mean values; *Welch two sample $t$-test; ${ }^{\star \star}$ Exact Wilcoxon rank sum test; ${ }^{* \star \star}$ Fisher exact test; SD: standard deviation.

thermore, no prolongation towards longer vaginal birth periods was seen in cases where magnesium sulfate was intravenously administered $(P=0.56)$.

No significant differences in adverse maternal outcome were observed between both study groups. In contrast, some fetal outcome variables differed between both groups: during labor, one case of hyperstimulation syndrome was observed in the dinoprostone group. Lower Apgar scores $(<7)$ at five minutes were observed in the dinoprostone group versus the misoprostol group $(P<0.05$; Table 2). Similarly, more neonates of the dinoprostone group $(n=12)$ were admitted to the NICU, compared to the misoprostol group $(n=6, P=0.068)$. During labor, no difference was observed in the frequency of meconium stained amniotic fluid $(P=0.6)$.

\section{Discussion}

This study assessed the effectiveness of vaginally administered misoprostol versus dinoprostone in pregnant women with preeclampsia and mostly unfavorable cervix [9]. The data show an association with the applied PG and delivery within $48 \mathrm{~h}$, supporting the results of our and other previous studies in the case of non-preeclamptic patients $[3,6,16]$. However, we did not see an association between a ripe cervix (Bishop score $>6$ ) and delivery intervals in both groups, in contrast to other studies in healthy pregnant women showing high predictive values of high Bishop scores [18, 20].

Only few trials have assessed the potential role of misoprostol and dinoprostone in preeclampsia [9, 12]. Our data are in accordance with those demonstrating a higher effectiveness of misoprostol than dinoprostone in IOL in patients with preeclampsia. The literature referring to labor induction provides some evidence that the use of misoprostol tends to be more effective than dinoprostone for cervical ripening, especially in patients with an unfavorable cervix. However, their use is frequently associated with uterine hyperstimulation, meconium stained amniotic fluid and fetal heart tracing abnormalities [19]. Cardiotocographic abnormalities occurred frequently after the use of misoprostol [11]. In our study we did not note these findings, probably due to our dose regimens, except for one case of hyperstimulation syndrome in the dinoprostone group. Various doses of misoprostol have

Table 2 Maternal and fetal outcome parameters of both groups.

\begin{tabular}{|c|c|c|c|}
\hline \multirow[t]{2}{*}{ Variable } & \multicolumn{2}{|l|}{ Drugs } & \multirow[t]{2}{*}{$\mathrm{P}$} \\
\hline & $\begin{array}{l}\text { Misoprostol } \\
\mathrm{n}=51\end{array}$ & $\begin{array}{l}\text { Dinoprostone } \\
n=47\end{array}$ & \\
\hline Birth weight $(\mathrm{g})$, mean & 3215 & 3019 & $0.11^{*}$ \\
\hline Median (SD) & $3270(631)$ & $3215(779)$ & \\
\hline Blood loss (mL), mean & 448 & 470 & $0.72^{*}$ \\
\hline Median (SD) & $400(200)$ & $400(157)$ & \\
\hline Induction to delivery interval (h) mean & 32.3 & 50.3 & $0.2^{*}$ \\
\hline Median (SD) & $26.0(20.0)$ & $35.8(39.4)$ & \\
\hline Number of drug applications (SD) & $2.41(1.7)$ & $2.89(2.1)$ & $0.29^{\star \star}$ \\
\hline APGAR score $<7$ at $5 \mathrm{~min}(\mathrm{n})$ & 1 & 6 & $0.05^{\star \star \star}$ \\
\hline Admission to NICU (n) & 6 & 12 & $0.068^{\star \star \star}$ \\
\hline Meconium stained amniotic fluid (n) & 8 & 10 & $0.60^{\star \star \star}$ \\
\hline
\end{tabular}

*Welch two sample $t$-test, ${ }^{* *}$ Exact Wilcoxon rank sum test, ${ }^{* * *}$ Fisher exact test. 
been explored for IOL. Misoprostol at doses of $25 \mu \mathrm{g}$ seems to be as effective as $50 \mu \mathrm{g}$ in selected patients $[13,18]$.

Regarding the application route of misoprostol, one study compared $50 \mu \mathrm{g}$ of misoprostol orally and vaginally four hourly to a maximum of five doses [14]. The authors demonstrated that vaginal misoprostol has a faster action than the oral route in equivalent doses. However, more hyperstimulations and higher intervention rates for fetal distress were seen in the vaginal group, using the above mentioned dose regimen. Therefore, more studies are needed to assess the risk-benefit ratio for both application routes. Some data suggest that preeclampsia is associated with longer induction to delivery intervals [5]. Griffiths et al. reported a median value of the induction to delivery interval of $17.48 \mathrm{~h}$ versus $12.7 \mathrm{~h}$ in the control group, using 1 or $2 \mathrm{mg}$ of prostaglandin E2, dependening on the Bishop score. Compared to the study of Griffiths and co-workers, the values of the induction to delivery intervals in this study are 1.9-fold higher in the misoprostol group, and 2.9-fold higher than in the prostaglandin E2 group, respectively. A recent study concluded that preeclampsia is not an independent risk factor for failed inductions after administration of PGE2, in contrast to maternal weight, unfavorable cervix, or magnesium sulfate [10]. In the case of intravenous magnesium, we could not confirm a trend towards longer time to delivery, in agreement with other studies that did not show a negative effect of intravenous magnesium sulfate therapy on labor duration [17, 21]. In our study, vaginal delivery was achieved in $78 \%$ of the misoprostol group, confirming findings from previous studies using misoprostol in case of preeclampsia $[4,9,12]$. The lower rate of $64 \%$ in the dinoprostone group may be explained by a higher proportion of failed inductions. Eleven of a total of 17 cesarean sections in this group were performed due to failed induction. Furthermore, more neonates from the dinoprostone group were admitted to the $\mathrm{NICU}$, either due to meconium aspiration (three of 12 cases), pneumonia (one case), hyperbilirubinemia (one case), preterm delivery (three cases), and delayed adaptation (four cases), compared to three neonates in the misoprostol group with slow adaptation, two with preterm delivery and one with a transient infant distress syndrome. Whereas dinoprostone is approved in most countries as vaginal suppositories or vaginal gel for IOL, misoprostol is not. However, numerous randomized studies exist on the use of misoprostol for induction of labor, and large specialist societies such as the ACOG recommend its use [1]. Nevertheless, the clinician has to take into account the medical and legal implications of off-label use of misoprostol in pregnant women.

In conclusion, our data indicate that vaginal misoprostol seems to be superior compared to prostaglandin E2 for cervical ripening and IOL in patients with preeclampsia, due to improved efficacy, a reduced need for CS due to failed IOL, a favorable side-effect spectrum and lower costs of the drug.

\section{References}

[1] ACOG Committee Opinion: Number 283, May 2003. New U.S. Food and Drug Administration labeling on Cytotec (misoprostol) use and pregnancy. Obstet Gynecol. 2003; 101:1049-50.

[2] Clinical effectiveness support unit. The use of electronic fetal monitoring. The use of cardiotocography in intrapartum fetal surveillance. Evidence-based clinical guideline number 8. London: RCOG Press; 2001.

[3] Danelian P, Poter B, Ferri N, Summers J, Templeton A. Misoprostol for induction of labour at term: a more effective agent than dinoprostone gel. Br J Obstet Gynaecol. 1999;106:793-7.

[4] Del Valle GO, Sanchez-Ramos L, Jordan CW, Gaudier FL, Delke I. Use of misoprostol to expedite delivery in severe preeclampsia remote from term. J Matern Fetal Med. 1996;5:39-40.

[5] Griffiths AN, Hikary N, Sizer AR. Induction to delivery time interval in patients with and without preeclampsia: a retrospective analysis. Acta Obstet Gynecol Scand. 2002;81: 867-9.

[6] Hofmeyr GJ, Gulmezoglu AM, Alfirevic Z. Misoprostol for induction of labor: a systematic review. $\mathrm{Br} \mathrm{J}$ Obstet Gynaecol. 1999;106:798-803.

[7] Hofmeyr GJ, Gulmezoglu AM. Vaginal misoprostol for cervical ripening and induction of labour. Cochrane database Syst Rev 2003 CD000941.

[8] Mercer BM. Induction of labor in the nulliparous gravida with an unfavorable cervix. Obstet Gynecol. 2005;105: 688-9.

[9] Nahar S, Rasul CH, Sayed A, Azim A. Utility of misoprostol for labor induction in severe pre-eclampsia and eclampsia. J Obstet Gynaecol Res. 2004;30:349-53.

[10] Park KH, Cho YK, Lee CM, Choi H, Kim BR, Lee HK. Effect of preeclampsia, magnesium and maternal weight on labor induction: a retrospective analysis. Gynecol Obstet Invest. 2006;61:40-4.

[11] Ramsey PS, Meyer L, Walkes BA, Harris D, Ogburn PL Jr, Heise $\mathrm{RH}$, et al. Cardiotocographic abnormalities associated with dinoprostone and misoprostol cervical ripening. Obstet Gynecol. 2005;105:85-90.

[12] Sahin HG, Sahin HA, Surucu R, Guvercinci M. A study of intravaginal misoprostol for induction of labor in toxemia of pregnancy. Int J Gynecol Obstet. 2001;75:3-9.

[13] Sanchez-Ramos L, Kaunitz AM, Delke I. Labor induction with $25 \mu \mathrm{g}$ versus $50 \mu \mathrm{g}$ intravaginal misoprostol: a systematic review. Obstet Gynecol. 2002;99:145-51.

[14] Shetty A, Danielian P, Templeton A. A comparison of oral and vaginal misoprostol tablets in induction of labour at term. Br J Obstet Gynaecol. 2001;108:238-43.

[15] Sibai BM, Gordon T, Thom E, Caritis SN, Klebanoff M, McNellis D, et al. Risk factors for preeclampsia in healthy nulliparous women: a prospective multicenter study. The National Institute of Child Health and Human Development Network of Maternal-Fetal Medicine Units. Am J Obstet Gynecol. 1995;172:642-8.

[16] Surbek D, Boesiger H, Hoesli I, Pavic N, Holzgreve W. A double-blind comparison of the safety and efficacy of intravaginal misoprostol and prostaglandin E2 to induce labor. Am J Obstet Gynecol. 1997;177:1018-23.

[17] Szal SE, Croughan-Minihane MS, Kilpatrick SJ. Effect of magnesium prophylaxis and preeclampsia on the duration of labor. Am J Obstet Gynecol. 1999;180:1475-9. 
[18] Szczesny W, Kjollesdal M, Karlsson B, Nielsen S. Bishop score and the outcome of labor induction with misoprostol. Acta Obstet Gynecol Scand. 2006;85:579-82.

[19] Wing DA. Labor induction with misoprostol. Am J Obstet Gynecol. 1999;181:339-45.

[20] Wing DA, Tran S, Paul RH. Factors affecting the likelihood of successful induction after intravaginal misoprostol application for cervical ripening and labor induction. Am J Obstet Gynecol. 2002;186:1237-40.

[21] Witlin AG, Friedman SA, Sibai BM. The effect of magnesium sulfate therapy on the duration of labor in women with mild preeclampsia at term: a randomized, doubleblind, placebo-controlled trial. Am J Obstet Gynecol. 1997;177:1561-2.

[22] Xenacis EM, Piper J, Field N, Conway D, Langer O. Preeclampsia: Is induction of labor more successful? Obstet Gynecol. 1997;89:600-3.

Received September 25, 2006. Revised January 24, 2007. Accepted February 20, 2007. Published online March 22, 2007. 